Research Article

\title{
Contact and Noncontact Ultrasonic Nondestructive Test in Reinforced Concrete Beam
}

\author{
Jason Maximino C. Ongpeng $\mathbb{D}^{1}{ }^{1}$ Andres Winston C. Oreta, ${ }^{2}$ and Sohichi Hirose ${ }^{3}$ \\ ${ }^{1}$ Associate Professor, De La Salle University, 2401 Taft Avenue, 1004 Manila, Philippines \\ ${ }^{2}$ Professor, De La Salle University, 2401 Taft Avenue, 0922 Manila, Philippines \\ ${ }^{3}$ Professor, Tokyo Institute of Technology, W8-22, 2-12-1, Ookayama, Meguro-ku, Tokyo 152-8552, Japan \\ Correspondence should be addressed to Jason Maximino C. Ongpeng; jason.ongpeng@dlsu.edu.ph
}

Received 26 July 2018; Revised 4 October 2018; Accepted 16 October 2018; Published 1 November 2018

Academic Editor: Flavio Stochino

Copyright ( 2018 Jason Maximino C. Ongpeng et al. This is an open access article distributed under the Creative Commons Attribution License, which permits unrestricted use, distribution, and reproduction in any medium, provided the original work is properly cited.

\begin{abstract}
Contact-type ultrasonic test is commonly used in construction industry where gel-couplant is applied to the material being tested and the transducers to assure that wave propagation will travel through without any air gaps. However, this method has disadvantages, since surface preparation is considered prior to testing. Another method of testing without the worry of air gaps that causes scattering of waves before it reaches the medium is the use of the noncontact ultrasonic test. In particular, the aircoupled ultrasonic test is done in this paper for reinforced concrete beams. Sixteen plain concrete cube specimens under the compression test and six reinforced concrete beam specimens under the four-point bending test are made with water-cement ratio of $40 \%$ and $60 \%$. The plain concrete cubes are investigated to establish the relationship of the contact ultrasonic test and load. Added parameters are considered to investigate the sensitivity of the contact and noncontact ultrasonic test in reinforced concrete beams. These are ultrasonic wave path and the neutral axis index. It shows that the higher water-cement ratio produces good sensitivity in the noncontact ultrasonic test, since it produces more cracks on the tension face. Lower water-cement ratio gives good sensitivity with load for the contact ultrasonic test, since it has its ultrasonic wave path passing through the concrete experiencing compression. In addition, the neutral axis index for a member subjected to bending is an important factor in assessing the sensitivity of both contact and noncontact ultrasonic test.
\end{abstract}

\section{Introduction}

Structures need to be assessed using structural health monitoring techniques especially for the predominant materials like reinforced concrete. Concrete comprises water, cement, sand, and aggregates with some admixtures that bond together to form a porous material. This heterogeneous material is very complex due to its nonlinear characteristics. Concrete proves to be a challenge in the field of nondestructive methods compared to metal.

One effective way of testing materials is the use of the ultrasonic test. Combination of the ultrasonic test using ultrasonic pulse velocity (UPV) and rebound hammer is introduced to test on site strength of concrete [1]. Test combinations with UPV to improve the prediction of strength of concrete are still limited due to its insensitivity to the changes in load [2]. The conventional ultrasonic test uses transducers with gel-couplant applied on the material to assure that the ultrasonic wave in contact with the material being tested will have no air gaps. Presence of air gaps in the contact-type ultrasonic test is a disadvantage, since it can scatter the wave propagation that can arrive at low-sensitivity measurements. In actual field inspection, the contact ultrasonic test with gel-couplant is troublesome due to the accessibility of inspector to the concrete surface being investigated.

Another way of testing concrete without considering the air gaps is the use of the noncontact ultrasonic test. In particular, the air-coupled ultrasonic test is a good example to eliminate the use of gel-couplant which is a significant factor in data collection and analysis. Air-coupled sensors were developed in the 1970s and were mainly used for 
inspection of wood and quality control of paper, art objects, and advanced composite materials used in the aerospace industry [3]. Quality tests in paper focused on sound dispersion and attenuation and estimation of its surface roughness can be monitored $[4,5]$. In construction building materials, this method is now being developed for noncontact detection of surface waves in concrete [3]. Additionally, the impact response test in the composite material using the $\mathrm{C}$-scan technique is also being developed in diagnosing concrete [6-8]. Various studies including wooden panel painting investigation and refraction including damping of timber laminates are also done in previous researches $[9,10]$.

Air-coupled ultrasonic test is gaining popularity and advancement. Waves in metals and composite materials have been successfully detected through the application of sensors having frequency of $50 \mathrm{kHz}$ to $1 \mathrm{MHz}$. In testing concrete, a flat frequency response below $100 \mathrm{kHz}$ is ideal. Low-frequency range sensors detect leaky waves in concrete. It is also proven that the use of air-coupled sensors is not sensitive to surface conditions in concrete and is sensitive to the existence of cracks as waves propagate across it [3]. It can also assess material nonlinearity [11] including carbonation inside concrete materials [12]. As the noncontact test method enables fast scanning of large structures, the amplitude information that is obtained is dependable and consistent when it is transmitted to the solid material [13].

The received time-domain waveform is the common data retrieved in this test. The parameters that are measured in time-domain waveform are time of arrival and peak to peak amplitude. Time of arrival of an ultrasonic wave passing through a dense medium should be smaller than the porous or damaged material. Peak to peak amplitude is sensitive to scattering of ultrasonic waves passing through the medium. In addition, received time-domain waveform is converted to frequency-domain waveform using fast Fourier transform. The parameters under frequency-domain waveform consist of fundamental amplitude and higher harmonic amplitudes. Previous research show that plain concrete cubes with different load-loading patterns greatly influences the higher harmonics parameters [14]. Complexity in the damage level for concrete is recognized when different sizes of aggregates inside concrete are present [15]. From references, the UPV test is convenient in detecting flaws like corrosion inside concrete [16], but it is insensitive to the internal damage. In addition, for plain concrete cubes, the correlation of ultrasonic parameters to internal damage represented by changes in load was done using artificial neural network model, and it indicated that UPV has very weak correlation compared to peak to peak amplitude of the time-domain waveform [17]. Investigation of reinforced concrete beams was considered using the artificial neural network model. The results showed that the contact-type ultrasonic test using peak to peak amplitude as a parameter to detect damage was classified as long- and short-range sensitivity for WC40 and WC60, respectively. Long- and short-range sensitivity from the model developed can detect damage more than $20 \%$ and less than $20 \%$, respectively [18].

In this paper, peak to peak amplitude of time-domain waveform is used as a parameter to measure the damage.
Decrease in peak to peak amplitude is evident when there is a scattering of the ultrasonic wave upon loading. The experimental test involved two test methods for both watercement ratio (WC) 60 and 40. These are the compression test of sixteen cube specimens of size $150 \mathrm{~mm}$ on each side and six concrete beams under the four-point bending test. The compression test is established to show the sensitivity of the peak to peak amplitude using the contact ultrasonic test, while the four-point bending test is performed to show the relationship of the reinforced concrete beam's load, neutral axis index, and the peak to peak amplitude for both the contact and noncontact ultrasonic test.

\section{Plain Concrete Cubes under Compression}

Sixteen concrete cubes were casted having size of $150 \mathrm{~mm} \times$ $150 \mathrm{~mm} \times 150 \mathrm{~mm}$. Varying water-cement ratios of WC40 and WC60 were used to investigate the effect of the test with different water-cement ratios. The maximum size of aggregates used was $20 \mathrm{~mm}$, and the ratio of sand-total aggregate was $45 \%$. Table 1 shows the content of the ingredients. A universal testing machine was used, and each specimen was subjected to the compression test at their mature age of 28 days. The compressive strength of WC40 and WC60 was tested prior to the experiment and resulted to characteristic strength of $53 \mathrm{MPa}$ and $40 \mathrm{MPa}$, respectively. The rate of loading/unloading was $0.5 \mathrm{kN}$ per second with the load pattern (Figure 1). Four loading branches were considered having a percent load of $0 \%$ to $20 \%, 0 \%$ to $40 \%, 0 \%$ to $60 \%$, and $0 \%$ to $100 \%$, respectively.

Specimens were tested by uniaxial compressive load (Figure 2(a)). Pitch-catch ultrasonic test was used to record the time-domain waveforms in each loading or unloading step. A high-gain broadband receiver and two transducers connected to the oscilloscope with tone-burst pulser were used in the setup. The supplied voltage was set at 1800 volts with the sine wave having 10 cycles at $100 \mathrm{kHz}$. In addition, the low-pass filter was set at $3 \mathrm{MHz}$, and the high-pass filter was set at $50 \mathrm{kHz}$ with an input impedance of $50 \Omega$. Direct transmission was implemented in the wave path of the ultrasonic wave from the transmitting to the receiving transducers (Figure 2(b)). This wave path was passing through the concrete experiencing compression. The transmitting and receiving transducers were carefully aligned, centered, and bonded to the concrete cubes with gelcouplants. The transmitting transducer had its generating frequency at $100 \mathrm{kHz}$ that sent signal across the specimen. The receiving transducer, on the contrary, had $200 \mathrm{kHz}$ frequency and was placed on the opposite face. This frequency of transducers proved to be effective in measuring internal damage inside the concrete material $[14,15]$.

Time-domain waveforms were recorded for the first loading branch of $0 \%, 20 \%, 40 \%, 60 \%$, and $80 \%$ (Figure 3 ). It was observed that the peak to peak amplitude decreases with respect to increasing load. A damage level assessment is used in this paper by solving its normalized peak to peak amplitude in decibels as seen in Equation (1). It followed the concepts in electrical circuits which dissipated power from one state to the other. This parameter is used to measure 
TABle 1: Design mix of concrete.

\begin{tabular}{lccccccc}
\hline \multirow{2}{*}{ Type } & \multirow{2}{*}{ Maximum size of gravel $(\mathrm{mm})$} & \multirow{2}{*}{ W/C $(\%)$} & \multicolumn{4}{c}{ Unit quantity $\left(\mathrm{kg} / \mathrm{m}^{3}\right)$} \\
& & & Water & Cement & Sand & Gravel & Water-reducing agent \\
\hline WC40 & \multirow{2}{*}{20} & 40 & 169 & 422 & 702 & 1039 & 0.84 \\
WC60 & & 60 & 175 & 291 & 812 & 1021 & 0.58 \\
\hline
\end{tabular}

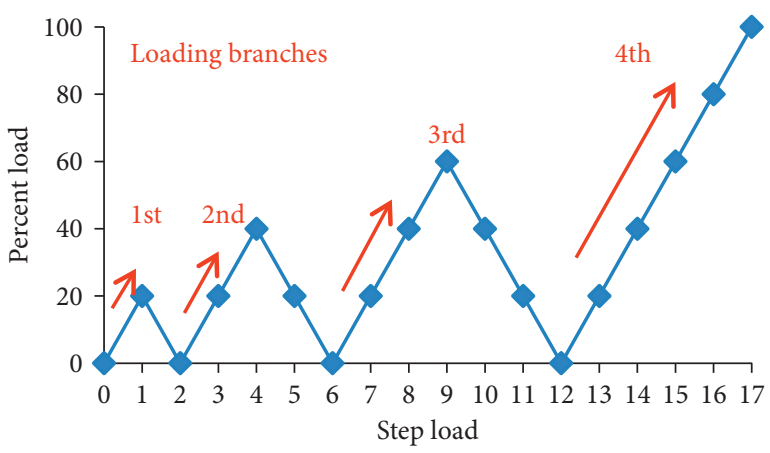

FIGURE 1: Multiple loading/unloading patterns for plain concrete WC40 and WC60.

damage presented when amplitudes decrease with respect to load $[14,15]$ :

normalized peak to peak amplitude $(\mathrm{dB})=20 * \log \left(\frac{A 1}{A 0}\right)$,

where $A 1$ is the peak to peak amplitude at any load (volts) and

$A 0$ is the maximum peak to peak amplitude throughout the test (volts).

The average normalized peak to peak amplitude of WC 40 and 60 is calculated (Figure 4). The damage level is inversely proportional to the load for both WCs. High WC produces larger magnitude of normalized peak to peak amplitude due to the air voids in the material that led to wide scattering of the ultrasonic wave when it passes the medium. The damage level for each loading branch L1 to L4 is recorded and compared (Figure 5). The magnitude of the damage is proportional to the load increase in the concrete cubes. The results gave good agreement for plain concrete when subjected to the ultrasonic test using peak to peak amplitude as a parameter to measure the load it experiences under direct transmission with the medium under compression.

\section{Reinforced Concrete Beams under the Four-Point Bending Test}

There were six $100 \mathrm{~mm} \times 100 \mathrm{~mm} \times 400 \mathrm{~mm}$ singlereinforced concrete beams specimen with WC of 60 and 40. The reinforcing bar used was $9 \mathrm{~mm}$ in diameter with a yield strength of $400 \mathrm{MPa}$ and was placed at the bottom of the beam with a concrete cover of $10 \mathrm{~mm}$ (Figure 6). The concrete design mix was the same as the plain concrete cubes in the previous section. A notch of $3 \mathrm{~mm}$ was made at the midspan of the bottom beam. This was to make sure that the crack formation monitored by the air-coupled ultrasonic test was locally focused at the concrete surface in tension. The use of $9 \mathrm{~mm}$ reinforcing steel bar parallel to the position of the transducers were assumed to be negligible. In the UPV test, with good quality concrete, the presence of the $12 \mathrm{~mm}$ diameter bar is insignificant [19].

Four-point bending test was done together with the aircoupled ultrasonic test. The air-coupled ultrasonic transducers (the transmitter and receiver) were oriented 8 degrees from the vertical axis and were focused on the midspan tension side where the bending/tension cracks occurred. The air-coupled ultrasonic test was focused on the inverted setup using the Japan Probe JPR-660C with the $200 \mathrm{kHz}$ aircoupled transmitter and receiver. Maximum voltage was set at $600 \mathrm{~V}$ with the burst wave of 10 waves.

It was made having an inverted layout (Figure 7) to properly place the air-coupled transducers upright along the midspan of the beam where tension crack occurred. Location of the strain gauges was placed at midspan and longitudinally along the beam positioned at the top and the bottom faces of the beam. The collection and analysis of data after the test was shifted to the upright position where the presence of moment in the beam was in the positive direction.

Loading and unloading paths were made to relate the behavior of the contact and noncontact ultrasonic test results' peak to peak amplitude with the load and the changes in its neutral axis index (Figure 8). There were 5 cycles of repeated load for $20 \%, 40 \%$, and $60 \%$, followed lastly by the final load until failure. The air-coupled ultrasonic transducers (the transmitter and receiver) were oriented 8 degrees from the vertical axis and were focused on the midspan tension side where the bending/tension cracks occurred (Figure 9). The air-coupled ultrasonic test was focused on the inverted setup, while the gel-coupled ultrasonic tested was oriented longitudinally under direct transmission (Figure 10).

The average normalized peak to peak amplitude of the three beams with WC60 is calculated using Equation (1) (Figures 11 and 12). For the noncontact ultrasonic test, the initial from step load 1 and 2 behaved inconsistent due to the initial state of the concrete before it stabilizes opening and closing of cracks according to the load applied. It can be noticed that the normalized peak to peak amplitude is sensitive to load especially from the step loads 31 to the end where it experiences loading and unloading from 0 to $60 \%$ of load. Incremental damage is seen to be consistent in each cycle of loading and unloading.

For the contact ultrasonic test with direct transmission along its length, the deviation of the normalized peak to peak showed sensitivity for load less than $40 \%$. However, it can be noticed that the measurement was not responsive to load since there were many cracks that caused a lot of scattering 


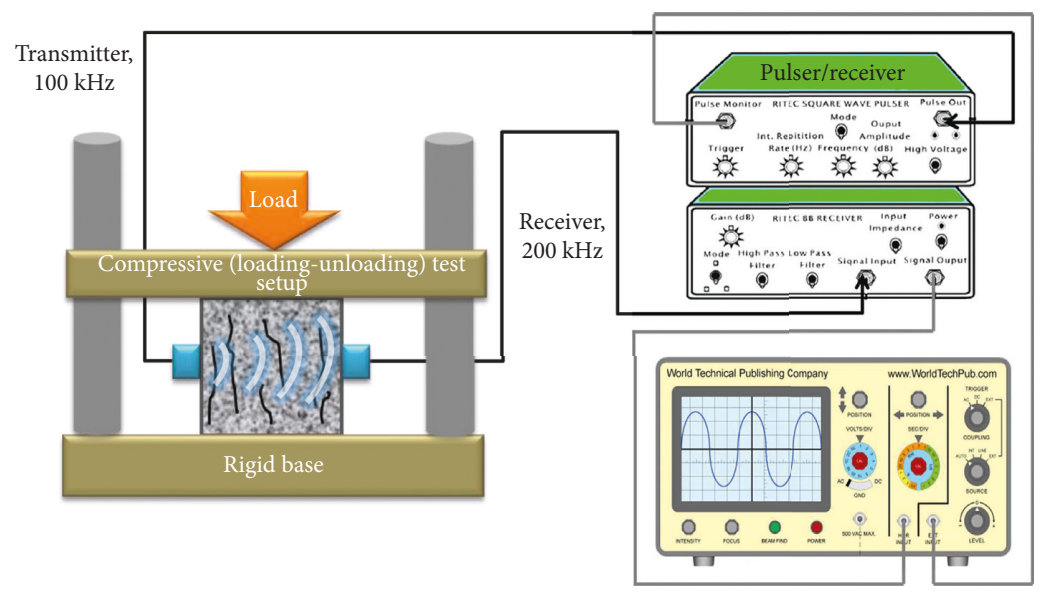

(a)

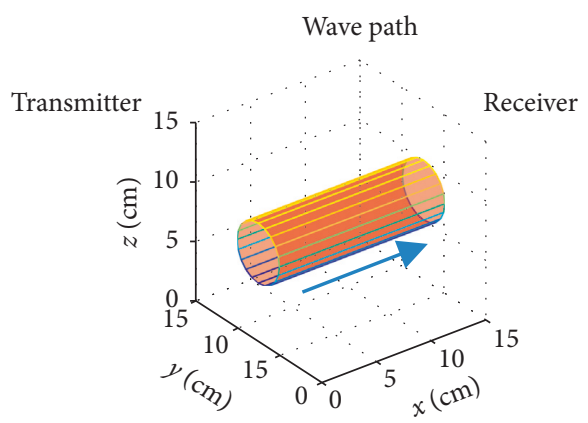

(b)

FIGURE 2: (a) Compressive testing procedure by loading and unloading for plain concrete with WC40 and WC60; (b) concrete cube showing ultrasonic wave path represented by a cylinder in direct transmission.

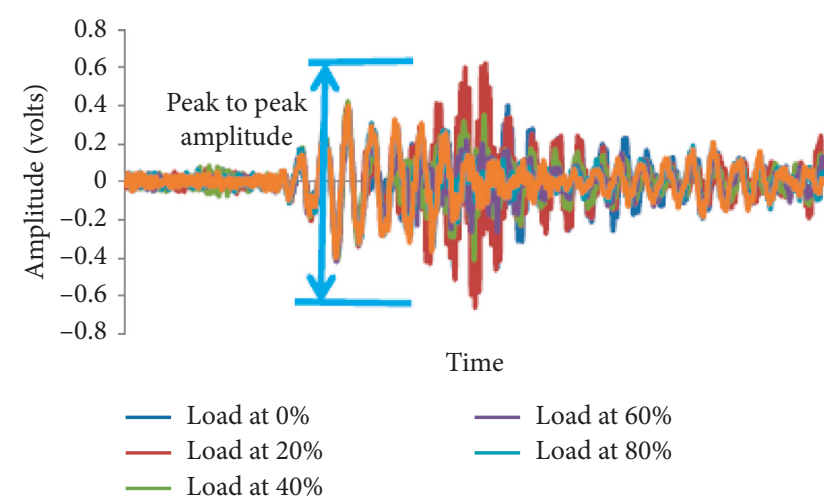

FIGURE 3: Time-domain waves showing peak to peak amplitude.

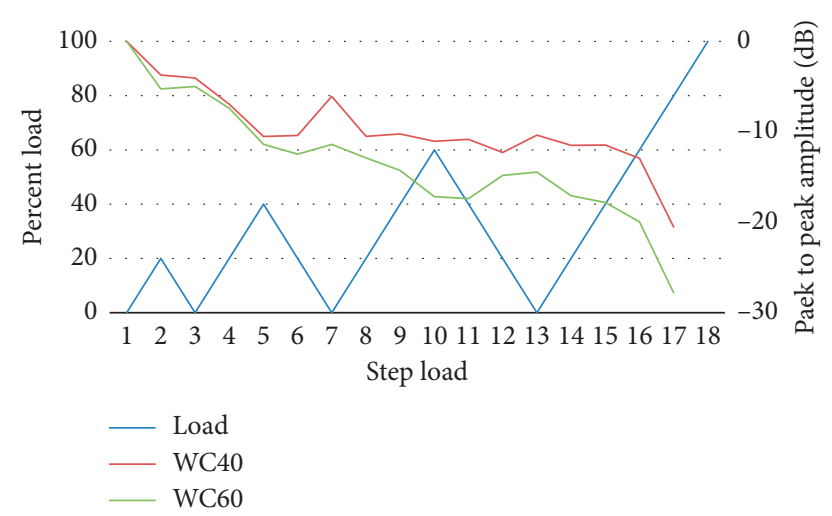

FIGURE 4: Total damage represented by normalized peak to peak amplitude.

inside the concrete after step load 13 ( $40 \%$ or more loads). Not responsive to load means that there was no significant resistance experienced in concrete or the fluctuations had no significant changes which is less than $1.5 \mathrm{~dB}$ from the undamaged state.

The behavior of damage for the contact and noncontact ultrasonic test is seen in Figure 12. The contact ultrasonic

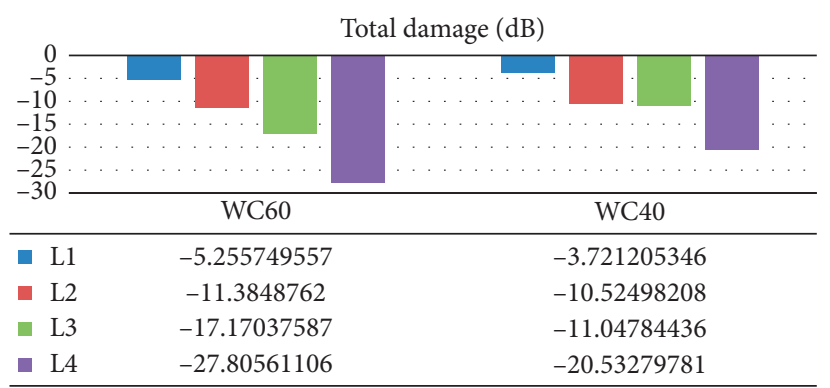

Figure 5: Total damage in each loading branch for plain concrete cubes under compression.

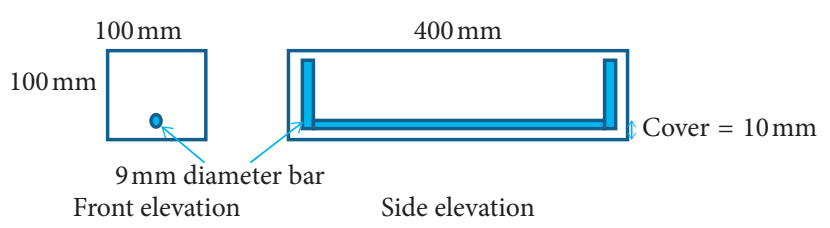

FIGURE 6: Reinforced concrete beam's front and side elevation.

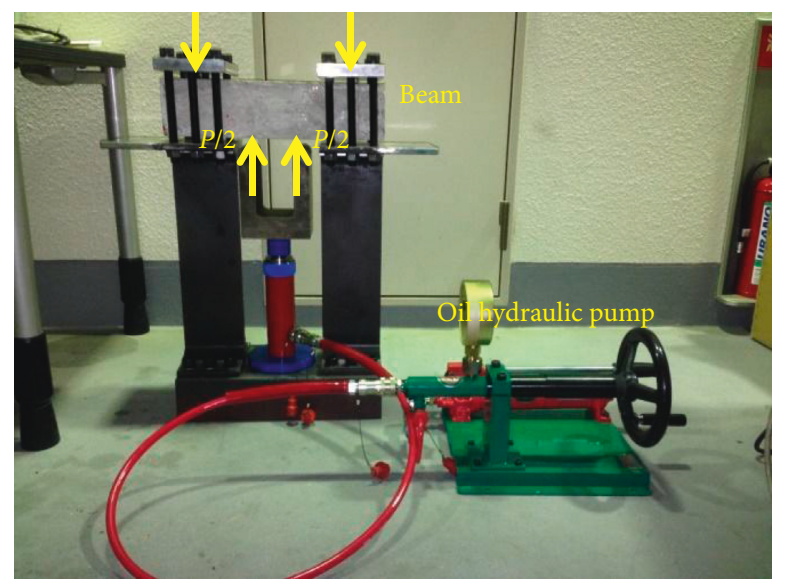

FIgURE 7: Reinforced concrete beam with WC40 and WC60 under the four-point bending test. 


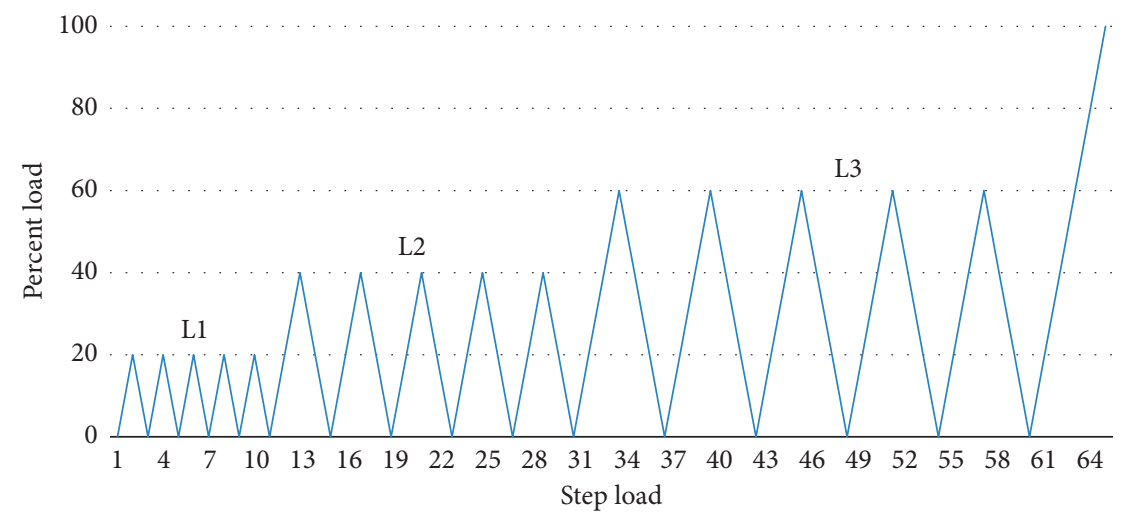

FIGURE 8: Multiple loading/unloading patterns for the reinforced concrete beam with WC40 and WC60.

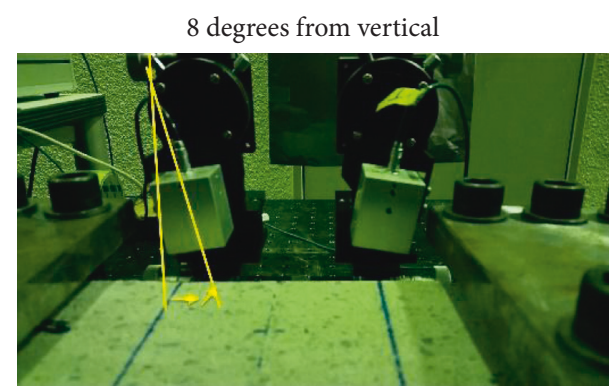

FIgURE 9: Air-coupled ultrasonic test setup.

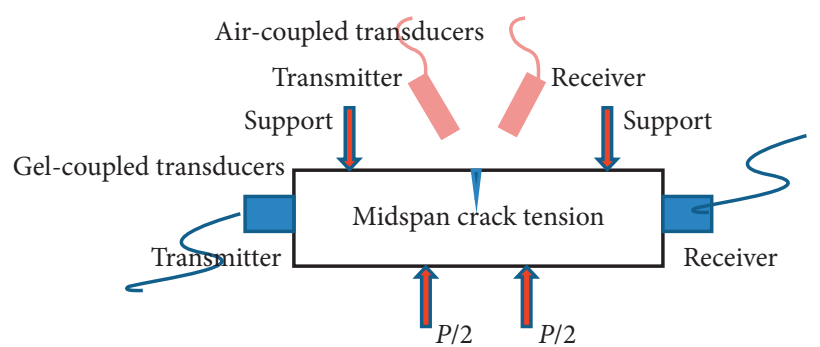

FIGURE 10: Contact and noncontact ultrasonic test performed in the reinforced concrete beam.

test behaved consistent with Section 2 for plain concrete cubes, whereas the normalized peak to peak amplitude decreases as load increases. On the other hand, the noncontact ultrasonic test was not responsive to load since there were many cracks that caused a lot of scattering inside the concrete after step load 13 ( $40 \%$ or more loads).

The complexity of the results (Figures 11 and 12) is brought about factors different from the plain concrete cube compression test results in the previous section. In the uniaxial compression test of concrete specimens, experimental results from literature showed that the ultrasonic pulse wave varied with the direction of compressive load. It was found out that UPV increases when there is an increase in compressive load for transducers placed parallel to the compressive load due to the acoustoelastic effect. Reduction of UPV occurred when the load is perpendicular to the transducers' placement [20]. Using the noncontact ultrasonic test, an increase in Rayleigh phase velocity was observed for concrete and mortar surfaces with the reduction of microcracks [21]. There are limited studies in the application of the ultrasonic test in reinforced beams whether it may focus on time-domain or frequency-domain waveforms. The flexural behavior of concrete is complex, since it comprises tension and compression zones with varying neutral axis index locations. In previous work, concrete slabs were tested using the four-point bending test. It was found out that the amplitude of the ultrasonic wave decreases in tension face and increases in compression face [22]. In addition, the UPV values increased when the bending load in the prestressed beam increases [23]. In the plain concrete cube, the contact ultrasonic test is done with the whole specimen experiencing compression, while the reinforced concrete beam experiences both compression resisted by concrete and tension resisted by the reinforcing bar. With this, another parameter is introduced, the neutral axis index. This parameter is promising in structural health monitoring where it is considered to be a sensitive universal parameter [24]. In determining the neutral axis index location with respect to changes in load in this paper, the recorded tensile and compressive strain is considered at the midspan of the beam. Diagram showing the cross section, sample strain diagram at a particular step load, and its corresponding stress diagram are considered (Figure 13).

This location of the neutral axis index is dynamic due to the changes in load that influences the strain at the top and bottom of the beam during the step loads in the experiment. The dynamic neutral axis index is computed using Equation (2). This location of the neutral axis index " $y$ " is correlated with the theory on the elastic design of reinforced concrete. In this study, the actual neutral axis index in the experimentation was very difficult to measure; hence, it was called the "neutral axis index" with the same formula shown in Equation (2):

$$
y=\frac{\varepsilon_{\mathrm{c}} h}{\left(\varepsilon_{\mathrm{t}}-\varepsilon_{\mathrm{c}}\right)},
$$

where $y$ is the neutral axis index in $\mathrm{mm}$,

$h$ is the height of the beam in $\mathrm{mm}$,

$\varepsilon_{\mathrm{c}}$ is the average strain at the midpoint top surface in compression, and

$\varepsilon_{\mathrm{t}}$ is the average strain at midpoint bottom surface in tension. 


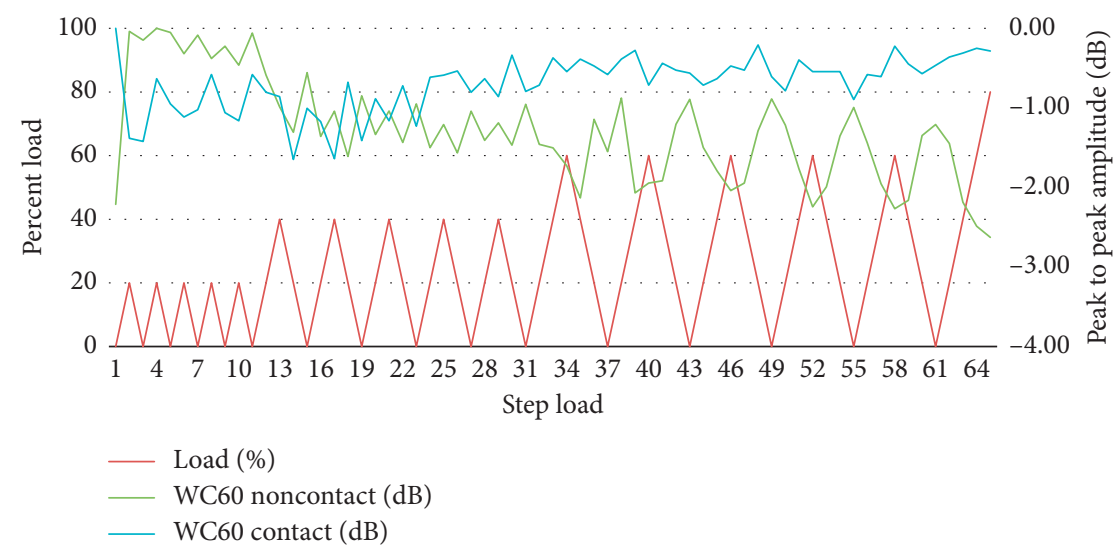

FIgURE 11: Average total damage represented by normalized peak to peak amplitude for WC60.

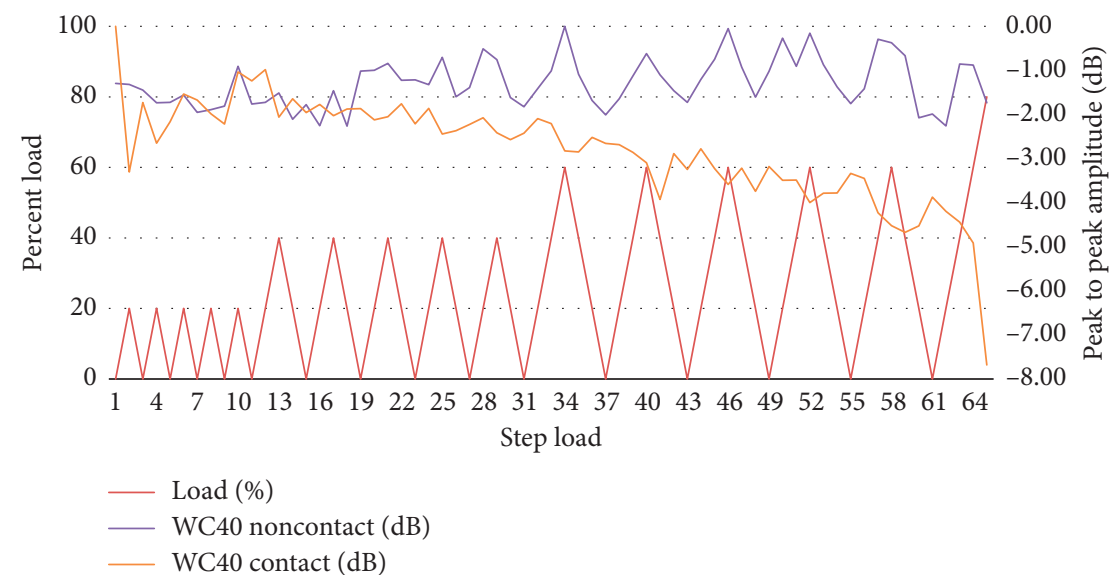

FIgURE 12: Average total damage represented by normalized peak to peak amplitude for WC40.

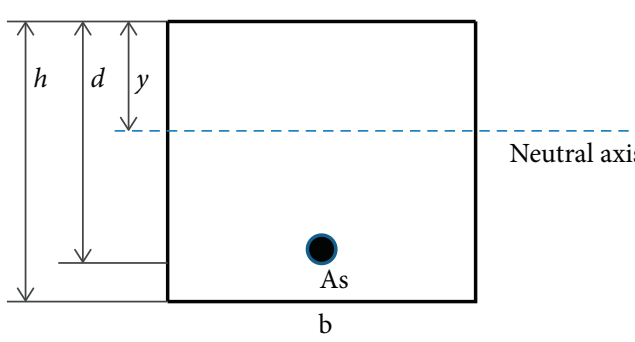

(a)

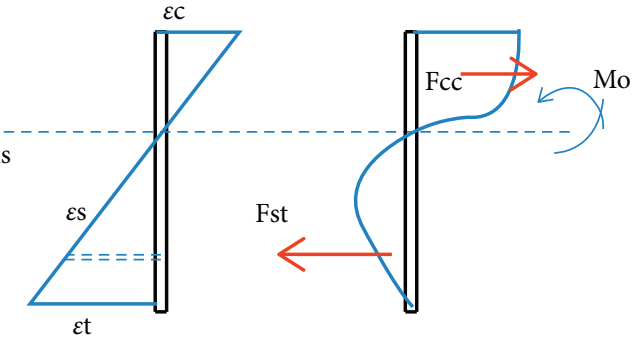

(b)

(c)

Figure 13: Neutral axis index across (a) cross section of the beam, (b) strain diagram, and (c) stress diagram.

The recorded strain data was smoothened and processed to determine the minimum and maximum peaks corresponding to each step load. Figure 14 shows the strain data of specimens 1, 2, and 3 with WC60. Specimens 1 and 2 were consistent with close standard deviation, while specimen 3 had minimal changes in its strain gauges at the latter loading branches. This was due to the limitation of using one strain gauge at the middle for each of the surfaces at the top and bottom where crack formation led to different patterns from one specimen to another. Furthermore, average of strain data was made to investigate the difference between WC40 and WC60, as seen in Figure 15. The average strain in tension and compression of WC60 was greater than that of WC40. The average tensile strain of WC40 produced relatively low values. This low value of average tensile strain at the beginning had experienced abrupt increase after it reached $60 \%$ of load where sudden crack formation was noticed visually during the test.

There were two theories to verify the behavior of the neutral axis index against the load: (1) the neutral axis index when the tension side of the concrete starts to crack, computed $y=49 \mathrm{~mm}$; (2) the neutral axis index when the 


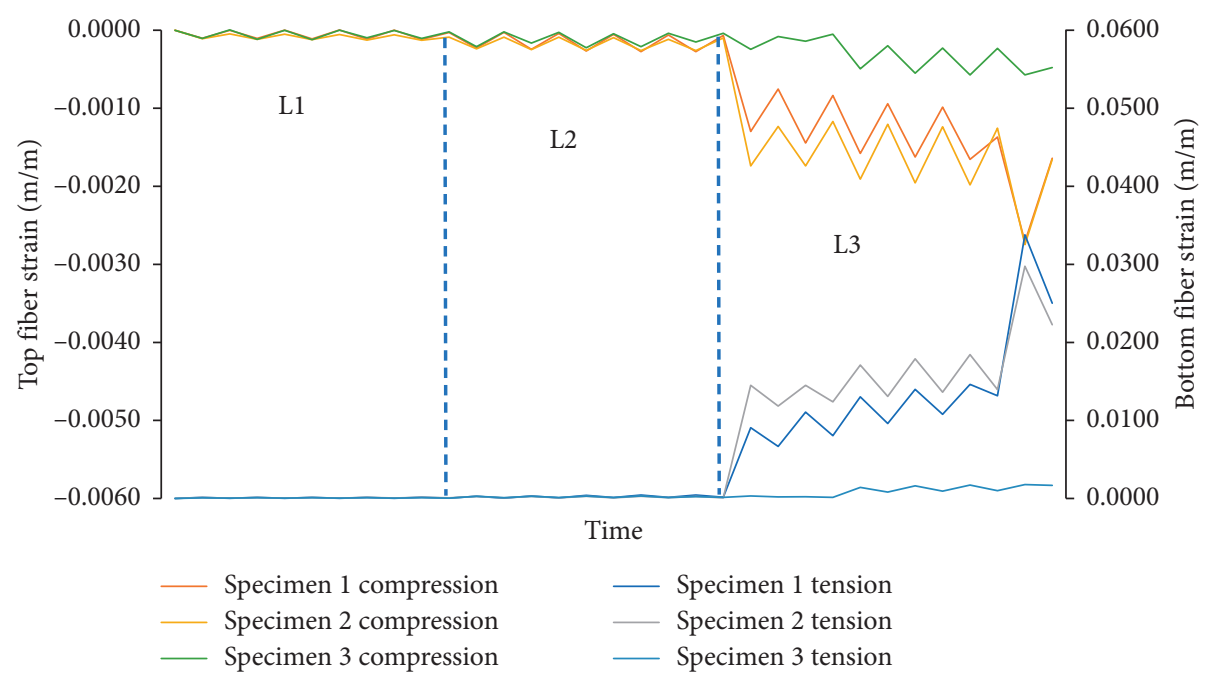

FIgURE 14: Strain data of specimens 1, 2, and 3 with WC60.

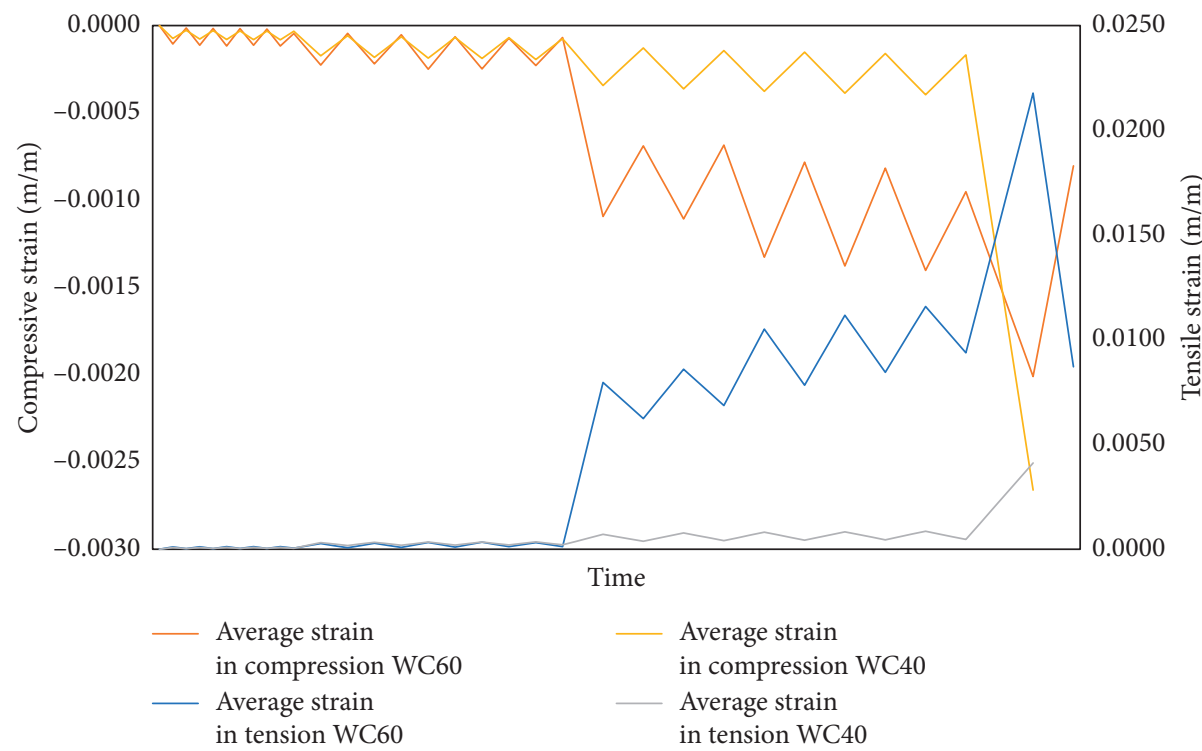

FIGURE 15: Average strain in tension and compression for WC60 and WC40.

concrete below is redundant, and only the reinforcing bar resists the moment in tension, computed $y=23 \mathrm{~mm}$ (Figure 16(a)).

In a reference, it was assumed that the $P$ wave or ultrasonic wave was perpendicular from the transducer's surface [25]. One factor that also affects the wave propagation is the orientation of fibers. This is important in determining the wave propagation in terms of wavefronts and ray path. It was found out that, for isotropic materials, the ray paths are collinear from the transmitter to the receiver with wavefronts perpendicular from the ray paths. Different behaviors occurred in the orthotropic material where acoustic wave propagation follows a curved ray path [26]. In this paper, the wave path was assumed to be the wave propagation from the transmitter to the receiver of ray paths and wavefronts that are inside the volume generated by the area of contact of the transducers from the transmission to the receiver. Any reflection and refraction outside the cylindrical volume was assumed to have a negligible effect on the data collection and analysis.

The wave path for the WC60 beam of the contact ultrasonic test was shown as the circular region covered by the wave path when the neutral axis index was $23 \mathrm{~mm}$ (Figure 16(b)). It can be noted that, during the L1 loading/unloading branches, the wave path passed through the compressive zone, and hence, sensitivity on the normalized peak to peak amplitude was experienced. However, in the L2 loading/unloading branch, normalized peak to peak amplitude was not sensitive due to lower concrete quality, where presence of more air voids was experienced after step load 13 (40\% or more load). Lastly, during the L3 loading/unloading branches, normalized peak to peak amplitude was not sensitive due to low concrete quality and that the ultrasonic wave path did not pass through the compression zone. Further investigation is recommended 


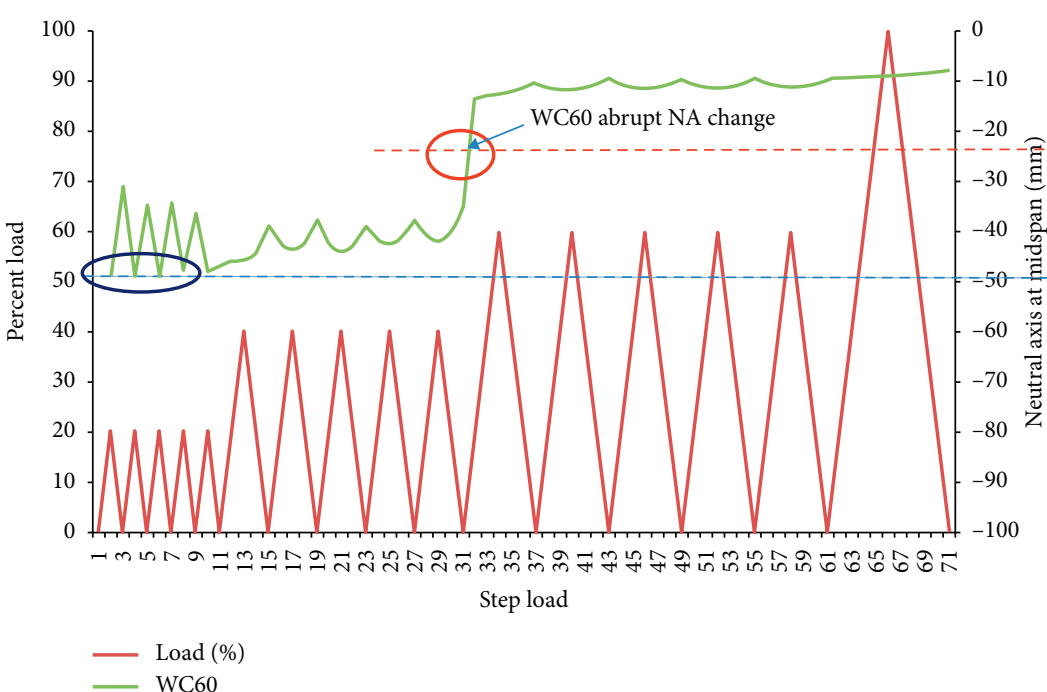

(a)

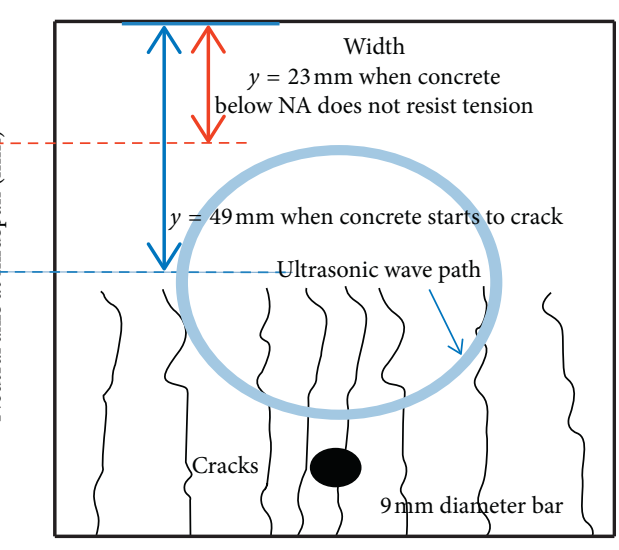

(b)

FIGURE 16: (a) Changes in the neutral axis index according to load for WC60; (b) cross section of the beam showing the ultrasonic wave path for WC60.

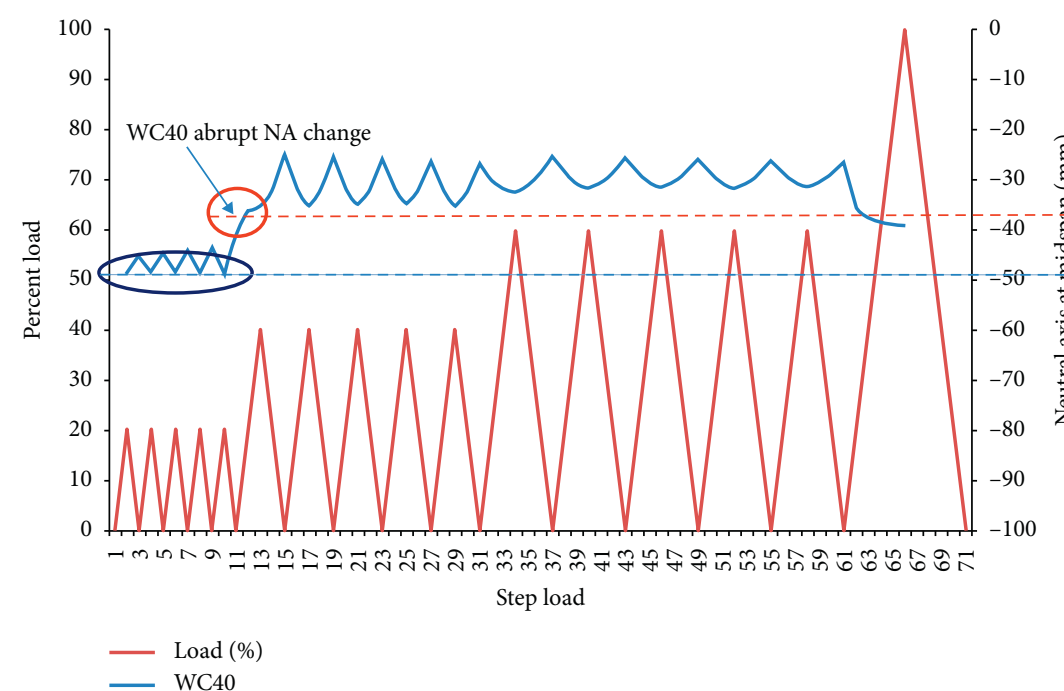

(a)

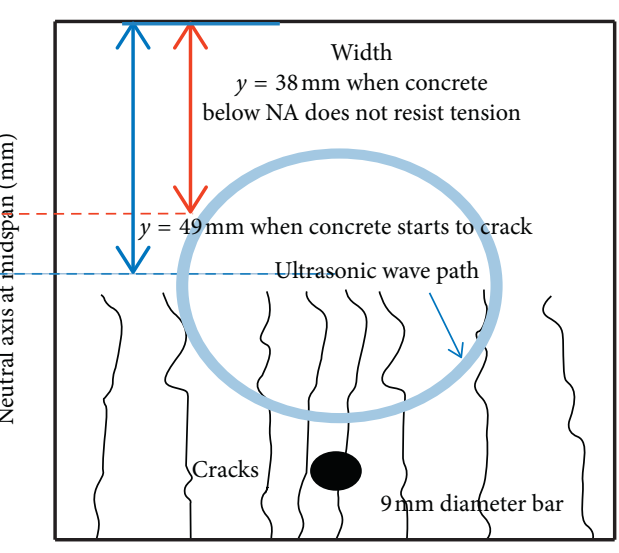

(b)

FIGURE 17: (a) Changes in the neutral axis index according to load for WC40; (b) cross section of the beam showing the ultrasonic wave path for WC40.

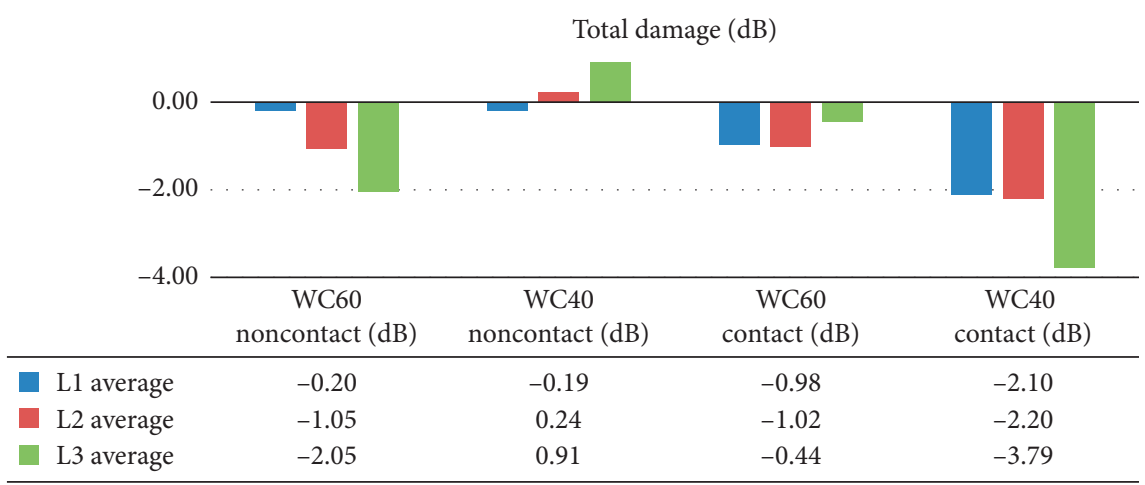

FiguRE 18: Total damage in each loading branch for reinforced concrete beams under the four-point bending test. 


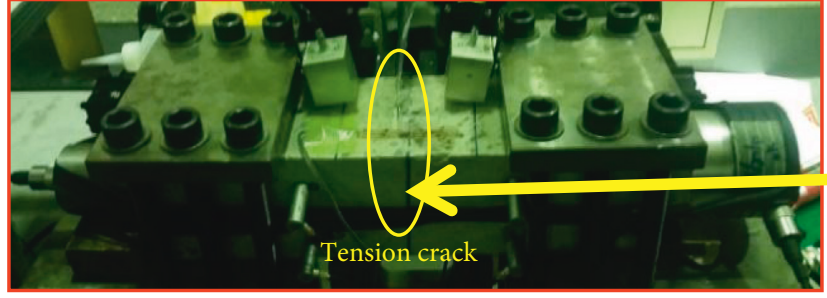

(a)

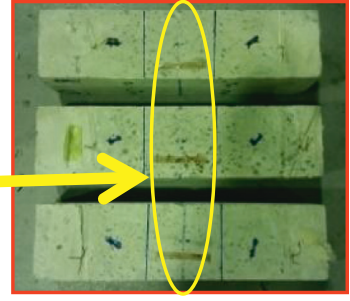

(b)

Figure 19: (a) Sample beam specimen picture during the test. (b) Pictures of tension cracks for the beam specimens.

using finite-element models to explore the interaction of ultrasonic waves inside low quality concrete with varying neutral axis locations. On the other hand, when the concrete below the neutral axis index provided significant cracks, the air-coupled ultrasonic test proved to be sensitive due to the opening and closing of cracks with the reinforcing bar holding the beam in place.

The neutral axis index when the tension side of the concrete starts to crack is computed having $y=49 \mathrm{~mm}$, while the neutral axis index when the concrete below is redundant and only the reinforcing bar resists the moment in tension is $38 \mathrm{~mm}$ (Figure 17(a)). The wave path intersects the compression zone experienced by the concrete that leads to better sensitivity of the contact ultrasonic test (Figure 17(b)).

The average total damage represented by the normalized peak to peak amplitude for high WC60 under the noncontact ultrasonic test and low WC40 under the contact ultrasonic test gave good agreement with the changes in load (Figure 18). Concrete with WC60 and WC40 under the contact and noncontact ultrasonic test, respectively, was not responsive. The actual crack formation on the tension side after the test was recorded (Figures 19(a) and 19(b)).

\section{Conclusions}

This paper focused on two types of specimens and testing under the nondestructive test. The plain concrete cubes tested with the contact ultrasonic test with varying WC of 40 and 60 under compression load showed that normalized peak to peak amplitude gave good agreement and was sensitive with the load. Higher water-cement ratio gave a larger value of normalized peak to peak amplitude. This was caused by the scattering of the ultrasonic wave inside the concrete, since it contained more air voids than lower watercement ratio. The second type of the specimen and test using reinforced concrete beams under the four-point bending test with varying $\mathrm{WC}$ of 40 and 60 proved to be complex. Added parameters were considered to investigate the sensitivity of the contact and noncontact ultrasonic test. These were ultrasonic wave path and the neutral axis index. It showed that the higher water-cement ratio produced good sensitivity in the noncontact ultrasonic test, since it formed more cracks. Lower water-cement ratio gave good sensitivity with load for the contact ultrasonic test, since it had its ultrasonic wave path passing the concrete experiencing compression. In addition, the neutral axis index for a member subjected to bending is an important factor in assessing the sensitivity of both contact and noncontact ultrasonic test. In future studies, nonlinear ultrasonic test using frequency spectrum and nonlinear finite-element model can be developed to show how the stress distribution with a varying neutral axis index is experienced. Variation of the formation of new cracks as well as closing and propagating of preexisting cracks can also be explored.

\section{Data Availability}

The data came from the dissertation of the corresponding author. The data are archived in Tokyo Institute of Technology.

\section{Conflicts of Interest}

The authors declare that they have no conflicts of interest.

\section{Acknowledgments}

The research has been made possible through the support of Japan Society for Promotion of Science (JSPS) RONPAKU.

\section{References}

[1] D. Breysse, "Nondestructive evaluation of concrete strength: an historical review and a new perspective by combining NDT methods," Construction and Building Materials, vol. 33, pp. 139-163, 2012.

[2] P. Daponte, F. Maceri, and R. S. Olivito, "Ultrasonic signalprocessing techniques for the measurement of damage growth in structural materials," IEEE Transactions on instrumentation and measurement, vol. 44, no. 6, pp. 1003-1008, 1995.

[3] J. Zhu and J. S. Popovics, "Non-contact NDT of concrete structures using air-coupled sensors," NSEL Report Series: Department of Civil and Environmental Engineering, University of Illinois, 2008.

[4] P. H. Brodeur, M. S. Hall, and C. Esworthy, "Sound dispersion and attenuation in the thickness direction of paper materials," Journal of the Acoustical Society of America, vol. 94, no. 4, pp. 2215-2225, 1993.

[5] J. Stor-Pellinen and M. Luukkala, "Paper roughness measurement using airborne ultrasound," Sensors and Actuators A: Physical, vol. 49, no. 1-2, pp. 37-40, 1995.

[6] K. Imielin, M. Castaings, R. Wojtyra, J. Haras, E. Le Clezio, and B. Hosten, "Aircoupled ultrasonic C-scan technique in impact response testing of carbon fibre and hybrid: glass, carbon and kevlar/epoxy composites," Journal of Materials Processing Technology, vol. 157-158, pp. 513-522, 2004.

[7] P. Purnell, T. H. Tan, D. A. Hutchhins, and J. Berriman, "Noncontact ultrasonic diagnostics in concrete: a preliminary 
investigation," Cement and Concrete Research, vol. 34, no. 7, pp. 1185-1188, 2004.

[8] J. Berriman, P. Purnell, D. A. Hutchins, and A. Neild, "Humidity and aggregate content correction factors for aircoupled ultrasonic evaluation of concrete," Ultrasonics, vol. 43, no. 4, pp. 211-217, 2005.

[9] A. M. Siddiolo, L. D’Acquisto, A. R. Maeva, and R. G. Maev, "Wooden panel paintings investigation: an air-coupled ultrasonic imaging approach," IEEE Transactions on Ultrasonics, Ferroelectrics and Frequency Control, vol. 54, no. 4, pp. 836-846, 2007.

[10] S. J. Sanabria, R. Furrer, J. Neuenschwander, P. Niemz, and P. Schultz, "Analytical modeling, finite-difference simulation and experimental validation of air-coupled ultrasound beam refraction and damping through timber laminates with application to non-destructive testing," Ultrasonics, vol. 63, pp. $65-85,2014$.

[11] S. Thiele, K. Kim, J. Qu, and L. J. Jacobs, "Air-coupled detection of nonlinear Rayleigh surface waves to assess material nonlinearity," Ultarsonics, vol. 54, no. 6, pp. 1470-1475, 2014.

[12] G. Kim, J. Kim, K. Kurtis, L. J. Jacobs, Y. L. Pape, and M. Guimares, "Quantitative evaluation of carbonation of concrete using nonlinear ultrasound," Materials and Structures, vol. 49, no. 1-2, pp. 399-409, 2016.

[13] J. Zhu and J. S. Popovics, "Non-contact imaging for surfaceopening cracks in concrete with air-coupled sensors," Materials and Structures, vol. 38, no. 283, pp. 801-806, 2004.

[14] J. M. Ongpeng, A. W. Oreta, and S. Hirose, "Effect of load pattern in the generation of higher harmonic amplitude in concrete using nonlinear ultrasonic test," Journal of Advanced Concrete Technology, vol. 14, no. 5, pp. 205-214, 2016.

[15] J. M. Ongpeng, A. W. Oreta, S. Hirose, and K. Nakahata, "Nonlinear ultrasonic investigation of concrete with varying aggregate size under uniaxial compression loading and unloading," Journal of Materials in Civil Engineering, vol. 29, no. 2, article 04016210, 2016.

[16] J. M. Ongpeng, "Ultrasonic pulse velocity test of reinforced concrete with induced corrosion," ASEAN Engineering Journal Part C, vol. 6, no. 1, pp. 5-12, 2017.

[17] J. M. Ongpeng, M. Soberano, A. W. Oreta, and S. Hirose, "Artificial neural network model using ultrasonic test results to predict compressive stress in concrete," Computers and Concrete, vol. 19, no. 1, pp. 59-68, 2017.

[18] J. M. Ongpeng, A. W. Oreta, and S. Hirose, "Investigation on the sensitivity of ultrasonic test applied to reinforced concrete beams using neural network," Applied Sciences, vol. 8, no. 3, p. $405,2018$.

[19] BS 1881:203, Recommendations for Measurement of Velocity of Ultrasonic Pulses in Concrete, British Standards Institution, London, UK, 1986.

[20] K. F. Bompan and V. G. Haach, "Ultrasonic tests in the evaluation of the stress level in concrete prisms based on acoustoelasticity," Construction and Building Materials, vol. 162, pp. 740-750, 2018.

[21] G. C. W. Kim, J. Y. Kim, K. E. Kurtis, and L. J. Jacobs, "Aircoupled detection of nonlinear Rayleigh surface waves in concrete-application to microcracking detection," NDT \& E International, vol. 67, pp. 64-70, 2014.

[22] F. Moradi-Marani, P. Rivard, C. P. Lamarche, and S. A. Kodjo, "Evaluating the damage in reinforced concrete slabs under bending test with the energy of ultrasonic waves," Construction and Building Materials, vol. 73, pp. 663-673, 2014.

[23] G. J. Kim, S. J. Park, and H. G. Kwak, "Experimental characterization of ultrasonic nonlinearity under cyclic change of prestressing force using nonlinear ultrasonic spectroscopy," Construction and Building Materials, vol. 157, pp. 700-707, 2017.

[24] D. H. Sigurdardottir and B. Glisic, "The neutral axis location for structural health monitoring: an overview," Journal of Civil and Structural Health Monitoring, vol. 5, no. 5, pp. 703-713, 2015.

[25] Y. Y. Lim, S. T. Smith, and C. K. Soh, "Wave propagation based monitoring of concrete curing using piezoelectric materials: review and path forward," NDT and E International, vol. 99, pp. 50-63, 2018.

[26] L. Espinosa, F. Prieto, L. Brancheriau, and P. Lasaygues, "Effect of wood anisotropy in ultrasonic wave propagation: a ray-tracing approach," Ultrasonics, vol. 91, pp. 242-251, 2019. 


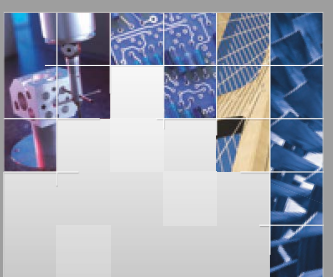

\section{Enfincering}
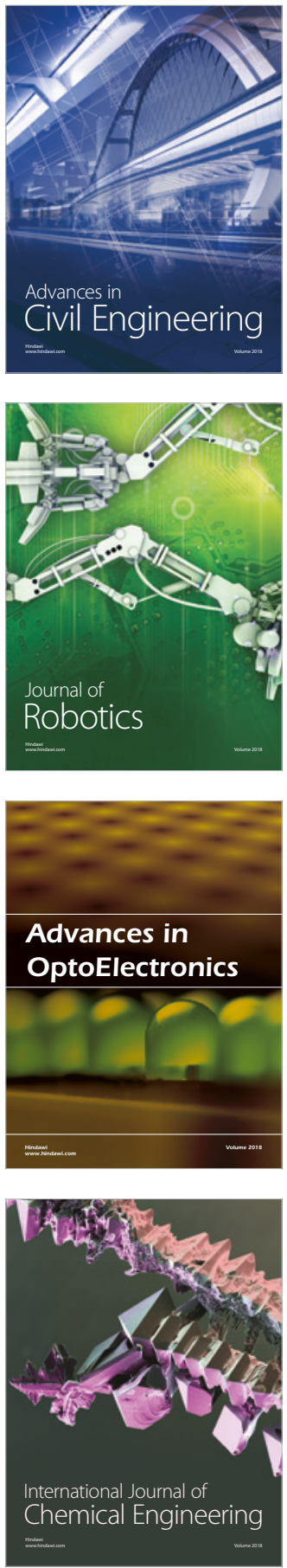

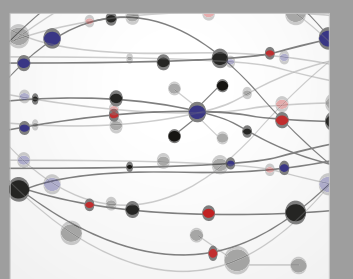

\section{Rotating \\ Machinery}

The Scientific World Journal

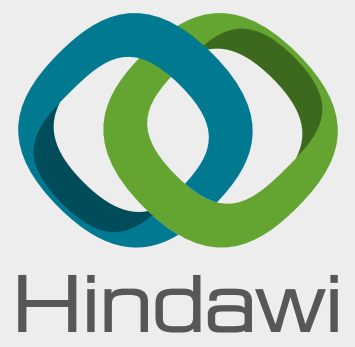

Submit your manuscripts at

www.hindawi.com
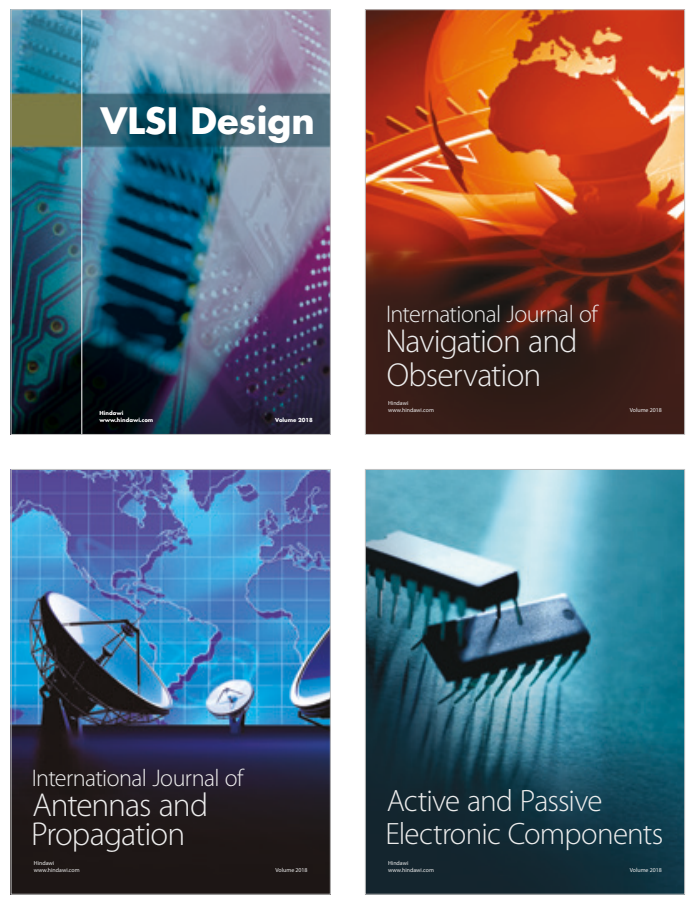
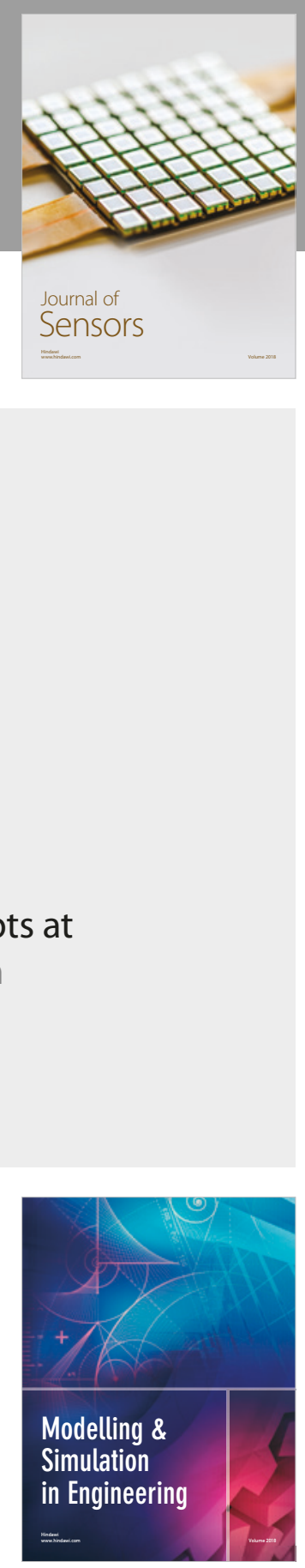

\section{Advances \\ Multimedia}
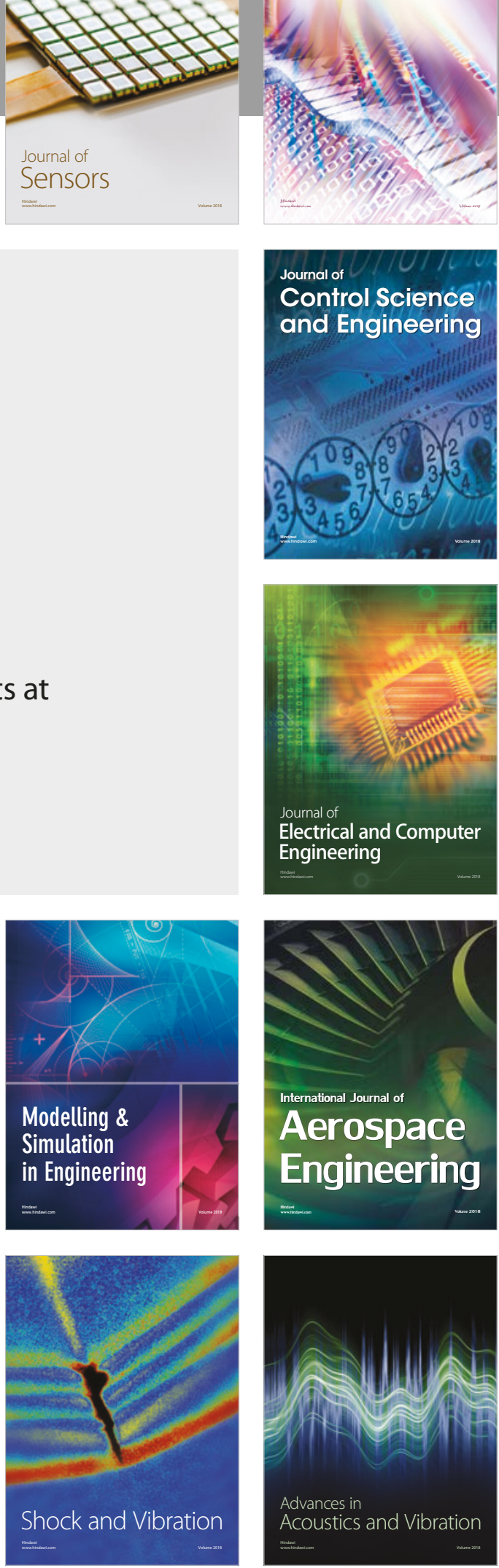